\section{$\underset{\substack{\text { hommes } \\ \text { \& migrations }}}{ }$}

\section{Hommes \& migrations}

Revue française de référence sur les dynamiques

migratoires

$1324 \mid 2019$

Religion et discrimination

\title{
1905 à l'ombre des « faiblesses humaines »
}

\section{Mustapha Harzoune}

\section{OpenEdition \\ Journals}

\section{Édition électronique}

URL : https://journals.openedition.org/hommesmigrations/8596

DOI : 10.4000/hommesmigrations.8596

ISSN : 2262-3353

\section{Éditeur}

Musée national de l'histoire de l'immigration

\section{Édition imprimée}

Date de publication : 1 janvier 2019

Pagination : 208-211

ISBN : 978-2-919040-44-5

ISSN : $1142-852 X$

\section{Référence électronique}

Mustapha Harzoune, « 1905 à l'ombre des « faiblesses humaines » , Hommes \& migrations [En ligne], 1324 | 2019, mis en ligne le 01 janvier 2019, consulté le 06 janvier 2022. URL : http:// journals.openedition.org/hommesmigrations/8596; DOI : https://doi.org/10.4000/ hommesmigrations.8596 


\section{Kiosque}

\section{5 à l'ombre des "faiblesses humaines 》 Mustapha Harzoune}

Sous peu, la calotte remplacera le sabre dans les bavardes messes télévisuelles. Après les mobilisations des gilets jaunes, commentées ad nauseam par tout ce que la France compte de képis et autres experts es maintien de l'ordre (manquait qu'Alexandre - pas le Grand - Benalla), sonnera bientôt l'heure de tout ce que la France compte de bondieuseries, en barrette, kippa, chéchia et hijab de bon aloi. Après les salamalecs et les jérémiades policiers, place à la componction et à la béatitude du curé et autre pasteur face à qui, on le sait depuis Sarkozy, l'instituteur républicain vaut peanuts! La France libertaire et légère, un brin anticlérical et antimilitariste, braillarde et paillarde, la France du Roman de Renard à celle de Brassens va manger son bonnet phrygien jusqu'à son dernier fil. Car nos sommités, élites de cabinet, fine fleur des cercles de l'entre-soi et autres manitous des think tanks de service, se sont découvert une nouvelle «occupation»; pardon, une nouvelle «responsabilité», «charge» «mission», un sacerdoce quoi! - au service du genre humain et du bien public (bien sûr): détricoter la sacrosainte loi de 1905. Loi qui ne doit plus seulement être de séparation des Églises et de l'État, mais aussi de la Mosquée et de l'État. Pourquoi cela, demande l'honnête béotien, le pedzouille, en gilet jaune ou pas? Histoire, dit-on dans le Landernau des "personnes bien informées», de couper l'herbe sous le pied des islamistes de tout poil et autres djihadistes, en tapant dans le porte-monnaie et en nettoyant la maison (française) de Mahomet, à commencer par son «clergé» autoproclamé, morcelé et incontrôlé, pseudopode d'États et d'organisations pas toujours bienveillants. Le porte-monnaie? «Limiter le financement des mosquées par des puissances étrangères [...] en facilitant le financement des associations cultuelles par la possibilité d'investir dans l'immobilier, et la possibilité de recevoir des aides de l'État "pour réparations et rénovation énergétique" » (Marianne, 14 novembre). Organiser le clergé? Entendre faire émerger " un interlocuteur unique, acceptable, modéré et capable de définir une ligne théologique compatible avec la culture française et l'état de l'opinion publique» (Yannick Prost, Sciences Po, The Conversation, 10 octobre). Et cela en s'impliquant peut-être dans la formation des imams. Quid alors non pas de la «neutralité» (comme dit Marianne), mais de la séparation - et une séparation qui donne la prééminence à l'État laïc (articles 25, 26, 30, 31 ou 35)? Pour Philippe Foussier, ancien Grand maître du Grand Orient de France: "C'est aux cultes de s'adapter aux règles républicaines, il n'y a pas de négociation ni de transaction à concevoir» (Marianne).

Ainsi, plutôt que de sérieusement fourrer son nez dans les financements saoudiens ou qataris de l'islamisme hexagonal, il est préférable de continuer à faire ses petites affaires avec «nos» amis, à commencer par MBS, Mohammed ben Salmane qui, après le meurtre du journaliste Jamal Khashoggi, fut accueilli à Tunis par le tonique hashtag "La ahla bika fi Tounès» soit «Tu n'es pas le bienvenu en Tunisie» ou par «Non à la profanation de la Tunisie, pays de la révolution» (Paris Match, le 26 novembre).

Ainsi, plutôt que de s'en remettre à l'efficacité des services de surveillance et de police, plutôt que de donner les moyens d'appliquer pleinement l'article 26 de la loi de 1905, plutôt que de non pas défendre, mais de rendre désirable ce qui fait le sel de ce pays et le commun de la société, on préfère "adapter la loi du siècle dernier à la poussée de l'intégrisme islamiste» (L'Opinion, 5 novembre).

À lire la presse et compte tenu de la panade du Conseil français du culte musulman (CFCM), dont « les dirigeants n'ont pas su conduire un dialogue satisfaisant avec les pouvoirs publics, ni prouver leur représentativité et leur capacité à se faire entendre des fidèles» (Y. Prost), on en arrive à souhaiter «bon courage » aux artisans de cette réforme. Pour autant, le vulgum pecus, majoritaire tout de même, abstème ou pas, n'a rien demandé. Rien que le droit à l'indifférence, le droit au partage, plein et entier, de ce qui fait la condition du citoyen français, laïc et républicain.

Pour les laïques, ce projet serait un «cassetête» car «comment assurer un meilleur contrôle des dérives radicales de l'islam sans remettre en cause la non-ingérence de la puissance publique dans les affaires religieuses»? II suscite 
déjà le «scepticisme: si certaines mesures de renforcement de police des cultes et de transparence financière s'envisagent parfaitement dans le contexte actuel, cela semble être présenté davantage comme une sorte de "deal" et donc de concessions mutuelles» pense Pierre Juston, doctorant en droit public et particulièrement remonté contre l'idée, qui trotterait dans quelques têtes, d'un concordat avec l'islam (Marianne, 14 novembre).

Les « responsables musulmans» seraient, eux, "inquiets de ce qu'ils perçoivent comme une intrusion des pouvoirs publics dans des questions qui touchent l'organisation de leur culte [et] ne cachent pas leur agacement, [concentrant] leurs critiques sur le consultant Hakim El Karoui, présenté dans les médias comme l'inspirateur du président de la République» (La Croix, 15 novembre). «Nous sommes unis, indépendants, légitimes et citoyens» clame Ahmet Ogras, le président du Conseil français du culte musulman (CFCM), "qui ne cache pas son "ras-le-bol des personnes qui parlent au nom de notre culte et qui ne représentent rien à nos yeux"».

\section{Entre les gros bras de Retailleau et le paradoxe de Burgat}

À droite, Bruno Retailleau dénonce "l'illusion dangereuse» en rappelant les «vaines tentatives» passées dont celle - chère à Sarkozy - d'un CFCM " lancé avec cette même volonté d'encadrer et de donner un visage à "l'islam de France". [...] En quoi, demande-t-il, est-il parvenu à lutter contre l'islamisme?» (Lepoint.fr, 16 novembre). «L'urgence aujourd'hui, c'est de renforcer la laïcité, pas de l'affaiblir.» Et de proposer «l'interdiction du voile islamique à l'université publique, la transformation du port de la burqa en délit avec suppression des allocations familiales pour les récidivistes, mais également d'inscrire dans la Constitution que nul ne peut se revendiquer de ses convictions religieuses pour se soustraire à la règle commune». Gaillard, il poursuit: «Expulsons les individus les plus dangereux, appliquons au salafisme les mesures pénales sur les dérives sectaires, renforçons notre arsenal juridique pour fermer les mosquées islamistes.» Il demande de «subordonner l'autorisation de financement étranger [...] à une série d'exigences [dont] le respect de la liberté religieuse et notamment la liberté de changer de religion». Arrive la grosse ficelle de l'immigration, utilisée le 10 décembre par le président lui-même lors de son allocution sur la crise dite des "gilets jaunes»: "Les enjeux de citoyenneté que pose le communautarisme islamique, écrit Retailleau, ne pourront être résolus sans traiter en profondeur les questions d'immigration, et sans renouer avec une conception généreuse mais exigeante de la citoyenneté française à travers l'assimilation.»

Le 10 novembre, sur un blog intitulé " "L'islam de France" de Hakim El Karoui: en arrière toute?», François Burgat, spécialiste aux positions souvent polémiques, fait la leçon à l'auteur du rapport qui aurait inspiré le projet d'organiser l'islam de France. Burgat dénonce «l'ostracisation d'une importante composante» des Musulmans de France, une gestion administrative et politique «dans la plus parfaite tradition coloniale» et cette conception qui veut que «la simple présence [de la religion] dans l'espace public [...] serait antinomique avec la "laïcité" et ne pourrait nourrir qu'une dynamique de dérives sectaires et radicales», sans oublier une représentation de «l'islam politique» figée dans «le ciment d'un immobilisme essentialiste». Burgat termine en pointant "un paradoxe essentiel des conditions de l' insertion" heureuse et efficace des Français de culture musulmane: pour réduire la fracture qui tend à les séparer de leurs concitoyens, engendrant le mal-vivre d'une grande majorité d'entre eux et les conduites de rejet violent d'un tout petit nombre, c'est particulièrement ceux qui s'expriment sur le registre oppositionnel qu'il faudrait inviter à participer davantage au processus de prise de décision en matière de "gestion de l'islam de France". Car ce sont justement ces voixlà qui font le plus défaut au concert citoyen et à l'alchimie de l'intégration"».

Voilà un projet de réforme qui laisse déjà remonter quelques remugles de la société française. Pourtant, comme l'écrit Yannick Prost, "s'il est nécessaire d'affaiblir l'influence extérieure de l'islam consulaire, il faut toutefois accepter la pluralité de l'islam en France, d'autant plus bienvenue que le premier mouvement ayant transcendé les origines nationales (UOIF, puis Musulmans de France) s'est d'abord caractérisé par une orthodoxie et une approche rigoriste en matière de mœurs». Quant à « la gestation» d'un «islam des Lumières», il «ne peut être conduit 
ni même suggéré par les autorités publiques». À tout le moins peuvent-elles «favoriser les conditions matérielles de la recherche et la formation des experts et des savants, sans s'abriter hypocritement derrière les principes de la loi de 1905. Il serait logique que le rayonnement de cet islam des lumières, lorsqu'il pourra jouer à armes égales avec les bataillons de théologiens formés et financés par nos amis saoudiens, puise sa source dans l'enseignement supérieur français». Deux «facteurs» expliqueraient, selon l'auteur, le succès de l'islam rigoriste: «l'exacerbation de l'orthodoxie [comme] moyen pour des ambitieux de conquérir une place dans l'espace social et politique» et, «préoccupant mais réversible: la revendication identitaire d'une jeunesse défavorisée ou déclassée, se cristallisant sur le marqueur religieux». "Apaiser la fièvre identitaire des quartiers, écrit Prost, ne peut se suffire d'un contre-discours, le traitement sera long et devra s'avérer global. Conforter un islam ouvert auprès des acteurs locaux, en respectant la démocratie dans l'espace privé qui est celui du religieux, en fera partie sans représenter l'essentiel de la solution».

\section{«Se présenter dans ses atours les plus rétrogrades"}

Mais quid de la laïcité à l'école demande RTL le 11 octobre, après la présentation par Jean-Michel Blanquer des «chiffres des atteintes à ce principe républicain en milieu scolaire»? Entre avril et juin 2018, un millier de signalements ont été recensés via l'adresse de saisine mise à disposition des enseignants - «ça en dit long sur les besoins d'accompagnement des collègues», soulignent les syndicats d'enseignants (Le Monde, 12 octobre). " 402 cas ont été traités [...] par les équipes dédiées des rectorats», dont soixante "jugés suffisamment "difficiles" pour nécessiter un déplacement de ces équipes dans les établissements concernés» (Le Monde). "Les signalements concernent à $57 \%$ les comportements d'élèves, $24 \%$ de parents et $8 \%$ de personnels de l'Éducation nationale» (RTL). « $44 \%$ concernent des collèges, $20 \%$ les lycées, et 36 \% (!) le premier degré» (Le Monde). La Dépêche du 11 octobre précise: " habits vestimentaires, certificats médicaux de complaisance, refus de se rendre à la piscine [...], refus de certains hommes de serrer la main des femmes, contestation au nom de la foi d'enseignements», comme les sciences ou la musique avec "des élèves qui se bouchent les oreilles». Pour Jean-Pierre Obin, «peu de choses ont changé» depuis son rapport de 2004 sur le sujet. Mais les chiffres publiés «ne sont sans doute que la partie émergée de l'iceberg [car] les enseignants sont très réticents à faire état de leurs difficultés». Iannis Roder, professeur à Saint-Denis constate, depuis 20 ans, "une pratique de la religion musulmane qui est devenue plus stricte, plus étendue, avec une forte pression pour respecter certaines règles alimentaires ou vestimentaires» (La Dépêche).

Le 25 novembre sur France Culture, Ghaleb Benchikh, en intitulant son émission «les provocations de la burqa et du burkini», donnait le ton: « la visibilité de la pratique islamique par certains courants radicaux pose un sérieux problème de vie dans la société française». Et de citer « les prières de rue, le port du voile intégral dans l'espace public et les affaires dites du burkini dans certaines plages». Il aurait pu ajouter à la piscine (Ouest France ou Le Télégramme, 26 septembre). Il y a le visible et, dans le secret des alcôves, ce qui l'est moins. Ainsi, « l'animateur de télévision égyptien Sayyed Ali recommande aux femmes d'apprendre le taekwondo pour se défendre contre les coups de leurs maris» (L'observatoire du Moyen-Orient, memri.fr, 21 novembre). Rappel: selon le juriste Ibn Nujaym, né en Égypte en 1520, le mari devra frapper son épouse dans les quatre cas suivants: si elle n'observe plus la prière quotidienne, si elle sort du domicile conjugal sans raison et sans son autorisation, si elle refuse de le rejoindre dans le lit alors qu'elle s'est purifiée des menstrues ou du retour de couches, si elle ne se fait pas belle après qu'il le lui a demandé (Catherine Kikuchi, The Conversation, 21 novembre). Il faut croire qu'avec ou sans Ibn Nujaym, les violences n'ont pas disparu. Le 2 mai 2014, dans Jeune Afrique, Fawzia Zouari, écrivaine et journaliste tunisienne, poussait un coup de gueule évoquant ces jours où elle «regrette d'être née arabe», notamment parce qu'elle se demandait «si c'était une manière de grandir l'islam que de le présenter dans ses atours les plus rétrogrades».

\section{Petits calculs}

Islamophobie? À voir. Car, pendant que certains hurlent à l'islamophobie, les mêmes se taisent sur 
Asia Bibi écrit Kamel Daoud (Le Quotidien d'Oran, 12 novembre). «Aujourd'hui, après son acquittement par la Cour suprême de ce pays, les islamistes paralysent le Pakistan, déferlent dans les rues, hurlent et se mobilisent.» S'il décide de parler d'Asia Bibi, c'est parce que "l'Algérie se "pakistanise" sous nos yeux» et qu'« alerter sur un crime à venir n'est pas criminaliser une religion mais ceux qui s'en servent». Il poursuit: "Soutenir cette femme aurait pu distinguer le musulman de l'islamiste, affirmer notre universalité, notre humanité et le souci de l'humain qui passe avant l'idéologie.» Mais voilà, "nos croyances passent avant notre humanité et surtout celle des autres. [...] C'est plus rentable de jouer les victimes que d'en défendre une. Plus facile. [...] La faute n'étant pas ce que nous avons fait de nous-mêmes et de notre monde, mais la faute est celle de ceux qui pensent mal de nous. Le miroir du monde devient coupable de notre nudité misérable. Alors on le casse ou on crie à l'islamophobie. Ou bien il faut écrire. Parler, dire et dénoncer nos propres misères et complicités». Écrire et dire, comme Aziz Chouaki, écrivain et dramaturge installé en France depuis 1991. À la fin des années 1980, "gandoura immaculée et barbe oblongue s'imposent comme la nouvelle norme. Le rock est mal vu et le Coran résonne dans les barres d'immeuble de son quartier populaire d'El-Harrach, "le 9-3 d'Alger", comme il dit». "Un jour, un copain me dit: "On a vu ton nom à la mosquée." J'étais sur la liste des gens à abattre. Je suis parti.» De Daoud ou de Sansal, il dit "respecter leur franc-parler" et "être souvent d'accord avec leurs idées", mais il les tacle sans ménagement [sic], estimant qu'ils "répondent à une attente du public français"». Ambiguïté de la réception! Lui-même "se sent mal compris au sein de l'intelligentsia parisienne, parfois réduit par certains à un immigré algérien rescapé du terrorisme». " "Quand j'écris sur autre chose que l'Algérie, on me dit que ça ne marchera pas. Et comme je ne chante pas les louanges de la femme musulmane opprimée par les islamistes, je ne suis pas à la mode en France", veut-il croire» écrivent Charlotte Bozonnet et Joan Tilouine (LeMonde, 25 septembre). Veut-il seulement le croire ou expose-t-il une autre et même réalité?

Pour en revenir à Dieu, il ne serait «rien d'autre que l'expression et le produit des faiblesses humaines» écrivait Albert Einstein dans une lettre vendue le 4 décembre à New York... 2,89 millions de dollars! N'est-ce pas ainsi qu'Allah est grand comme disait Alexandre Vialatte? 